\title{
MOLECULAR GENETICS OF A CILIATE
}

\author{
by
}

\author{
DAVID M. PRESCOTT
}

Department of Molecular, Cellular and Developmental Biology, University of Colorado, Boulder, Colorado 80309 USA

Keywords: DNA processing, gene-sized DNA, DNA replication, macronuclear development

The macronuclei of hypotrichs contains only gene-sized DNA molecules. About 24,000 different molecules are present, with an average copy number of 1,000 . All molecules have the same inverted terminal repeat sequence of 36 bases. The gene-sized molecules are created by excision from chromosomes during macronuclear development with destruction of spacer sequences between genes. Spacers represent $95 \%$ of the genome. The gene-sized molecules are individual replicons with DNA initiation occurring at or near one or both ends. Replication of the millions of molecules is achieved by replication machinery that traverses the macronucleus in a wave.

\section{INTRODUCTION}

HEINZ HOLTER delighted in the study of protozoa, particularly the large, free-living ameba. I worked with him at the Carlsberg Laboratory in 1954-55 both on amebae and Tetrahymena. Fashions have changed, and work on cells has become much more molecular, and the protozoa are as interesting as ever. In this paper I describe our work on a ciliated protozoan. The protozoa are a ceaseless source of surprises, and I think HEINZ will enjoy learning about the unique features of this particular beast.

Oxytricha nova is a common hypotrichous ciliate found in fresh water throughout the world. It makes a living by eating other ciliates, algae, and bacteria. Like all ciliates it has two kinds of nuclei (Figure 1). The micronucleus contains chromosomes, divides by mitosis, and undergoes meiosis during cell mating, but does not synthesize a detectable amount of RNA. The macronucleus contains much more DNA, divides by pinching in two (amitosis) and makes all the nuclear RNA needed for cell growth and reproduction and vegetative functions in general.

\section{Properties of macronuclear DNA molecules}

The DNA in the macronucleus is not organized into chromosomes, but instead occurs only in gene-sized molecules (11). Figure 2 shows macronuclear and micronuclear DNA by electron microscopy and Figure 3 shows macronuclear DNA distributed by size in an electrophoretic gel. The molecules range in size from 
400 base pairs (BP) in length to $15,000 \mathrm{BP}$ with a continuum of sizes in between. Each different sized molecule is a different gene. For example, the molecule at $\sim 1550 \mathrm{BP}$ is a gene for actin, and one at $1900 \mathrm{BP}$ is for $\beta$ tubulin. Genes for tRNAs, histones, and other proteins always occur in given sized molecules. The gene coding for $19 \mathrm{~S}, 26 \mathrm{~S}$, and 5.8S rRNAs is $7100 \mathrm{BP}$ long (12); it is differentially amplified compared to other genes, and the greater copy number is visible as a distinct band in an electrophoretic gel (Figure 3).

The number average size of macronuclear DNA molecules determined by electron microscopy is $2200 \mathrm{BP}$ (13). The total sequence complexity of macronuclear DNA is equal to $5.7 \times 10^{7} \mathrm{BP}(6)$. This means that there are about 24,000 different kinds of gene-sized molecules in the macronucleus. We accept this number as a tentative estimate of the number of genes in Oxytricha. The macronucleus contains $-57 \mathrm{pg}$ of DNA, which means that each of the 24,000 different molecules is present in 1,000 copies on the average. The DNA molecule coding for the three rRNAs is differentially amplified 100 -fold and is present in $10^{5}$ copies.

The 24 million DNA molecules in the macronucleus share an important structural feature. All of them have at each end the same nucleotide sequence in the following arrangement:
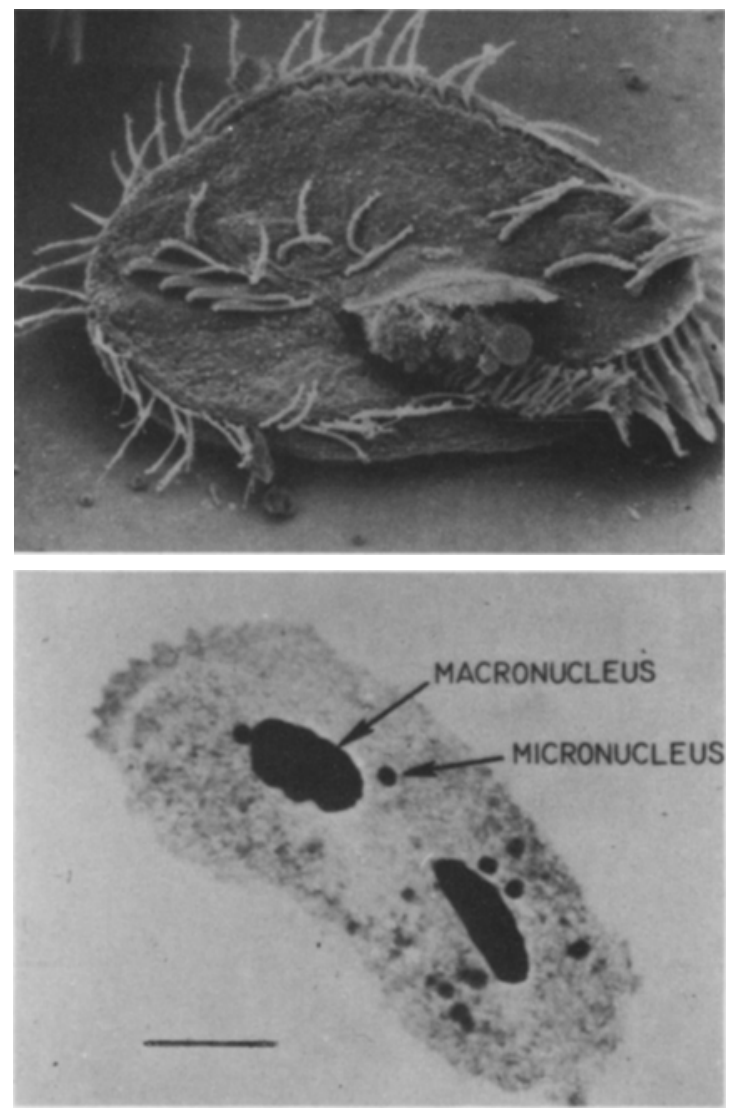

Figure 1. A scanning electron micrograph and a light microscope photograph (Feulgen staining) of the hypotrich Oxytricha nova. Two macronuclei and four micronuclei are characteristic of this species. Other stained material is food vacuoles. Bar $=30 \mu \mathrm{m}$.

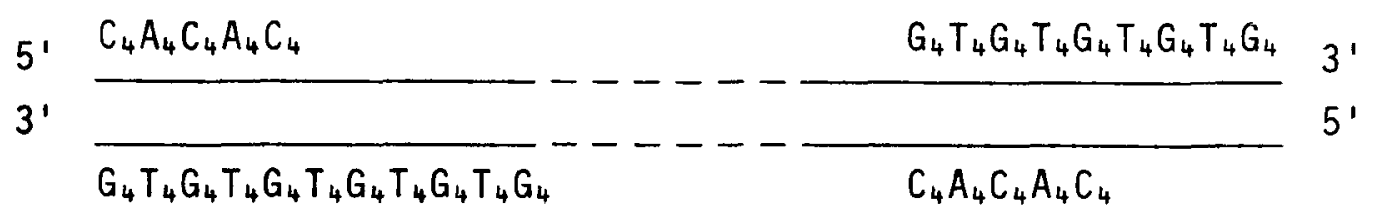

Each end has a single stranded 3' tail (or 5' gap) of 16 bases consisting of groups of guanine and thymine residues $(4,8)$. The alternation of groups of guanines and thymines continues for 20 more bases into the molecule and is matched in the 3' chain by groups of cytosine and adenine residues. This arrangement of inverted terminal repeats is not only highly conserved within a genus like Oxytricha but has been found in several kinds of distantly related genera. These termini are referred to as telomeres and are structurally similar to telomeres described for

yeast and Physarum chromosomes.

The telomeres confer in a way that remains a mystery the ability of the ends of linear DNA molecules to replicate. Remarkably, when the telomeres from Oxytricha molecules are joined to the ends of a linear piece of DNA, they provide functions that allow the molecule to replicate in yeast cells (9).

Telomeric structure and function have long puzzled geneticists and cell biologists. They are difficult to study because they represent such a minute amount of the total chromosomal DNA. 


\section{D.M. PrescotT: Ciliate DNA}

A diploid human cell contains 92 telomeres; on the average one telomere is present per $5 \times 10^{7}$ base pairs. In the macronucleus there are $48 \times 10^{6}$ telomeres or one per 1100 base pairs, and they constitute $3 \%$ of the total DNA. Obviously, the macronuclei of hypotrichs provide a uniquely favorable opportunity to analyze the structure, function, and significance of telomeres.
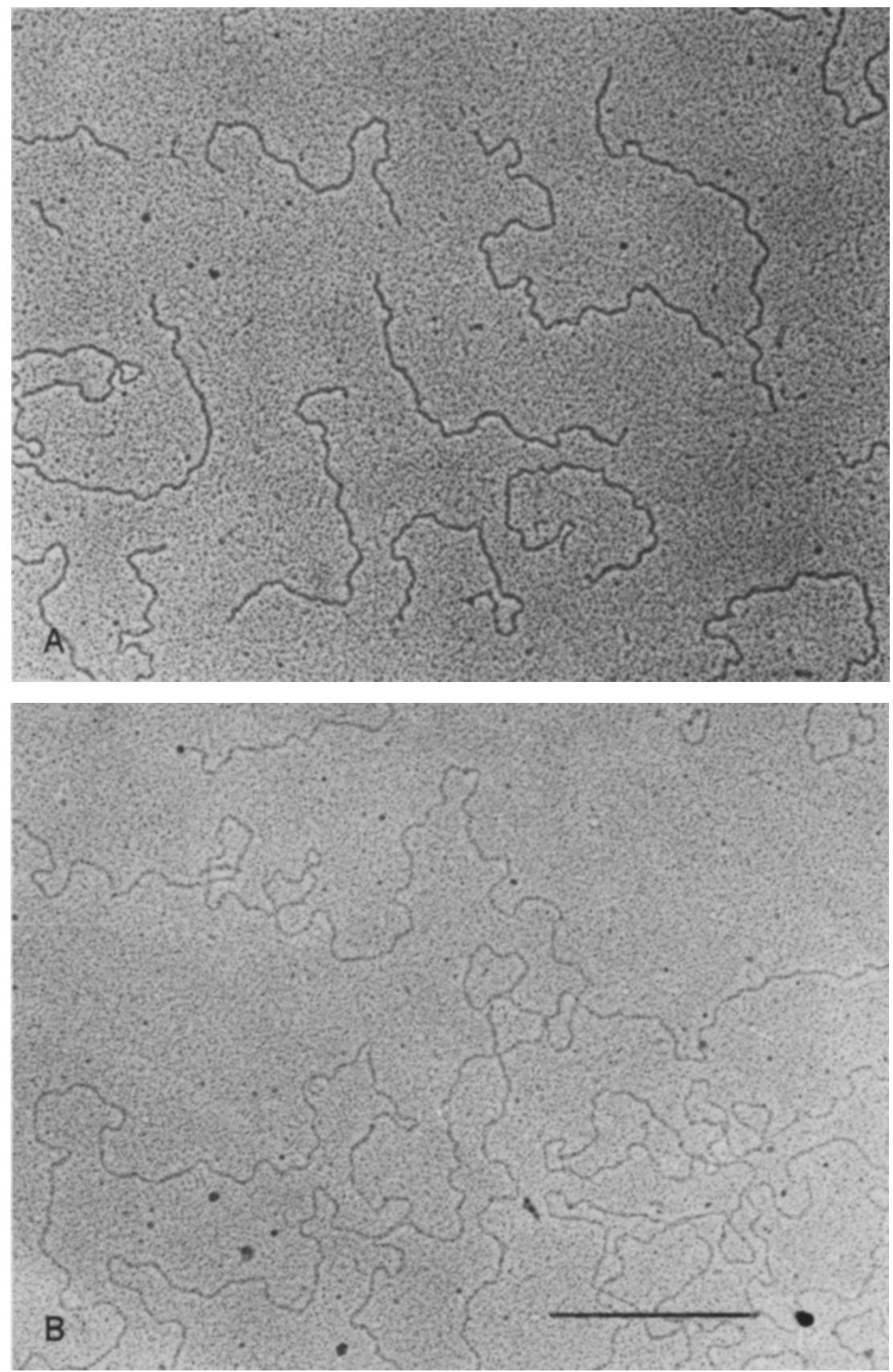

Figure 2. A) Gene-size, macronuclear DNA molecules from Oxytricha nova (by K.G. MURTI). B) A section of a DNA molecule from the micronucleus of Oxvtricha nova (by K.G. MURTI). Bar $=2.0 \mu \mathrm{m}$. 


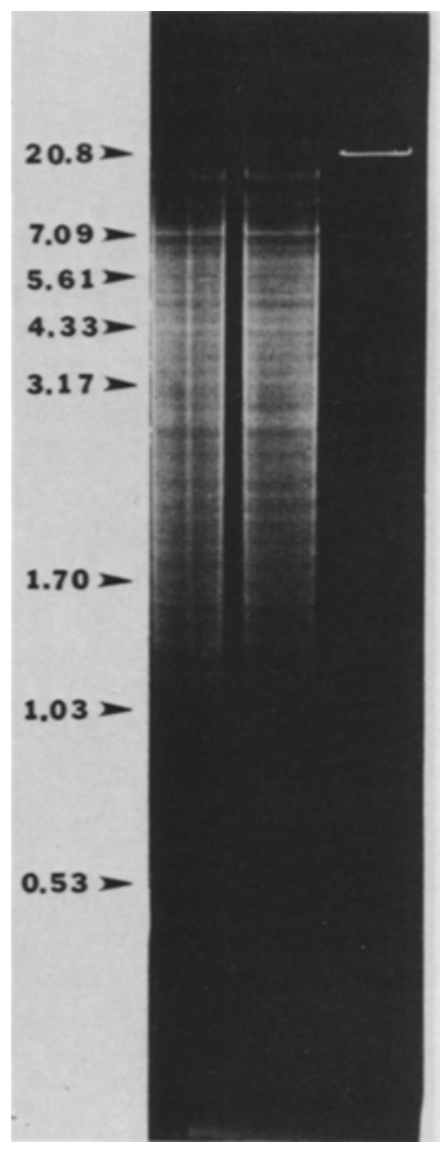

Figure 3. Macronuclear DNA from Oxytricha nova separated by size by electrophoresis on a $1 \%$ agarose gel. The two lanes to the left are for separate DNA preparations and are identical. The third lane (right) contains size markers. Sizes of DNA in kilobase pairs are marked on the far left.

\section{Replication of macronuclear DNA molecules}

Each of the 24 million DNA molecules in the macronucleus is a replicon that must be able to replicate independently. Replication in hypotrichs is achieved by means of replication machinery that is assembled at a point on the inner aspect of the macronuclear envelope. Species of Euplotes contain a highly elongated macronucleus, and the replication machinery is assembled at each end. The replication machinery travels through the macronucleus and is visible in the light microscope as a localized rearrangement of the chromatin, giving rise to a structure known as a replication band (Figure 4). The replication machinery in a band replicates each DNA molecule as it feeds into the band as the band travels through the macronucleus over several hours. In Oxytricha there is only one replication band that traverses the full length of the ovoid macronucleus. The two bands in Euplotes meet at the center of the elongated macronucleus where they disappear, signaling the end of DNA replication. Immediately thereafter the macronucleus rounds up and divides into two by amitosis in coordination with cytokinesis.

More structural detail of the replication band and of DNA synthesis can be seen by EM autoradiography. Figure 5 contains and EM autoradiograph of the macronucleus of a hypotrich. The replication band has two parts, a leading or forward zone identifiable by the regular, almost crystalline array of DNA-histone fibers. This organization disappears at the border between the forward zone and the rear zone. At this point the DNA becomes much diluted and replicates. The replicated molecules then spew out behind the rear zone where they are reorganized into the chromatin bodies typical of the macronucleus. Little is known about the molecular details of how a replication band works. One event of note is the turn off of RNA synthesis at the leading edge of the forward zone and its resumption at the rear edge of the rear zone.

Replicating forms of individual DNA molecules have been seen by EM. Some molecules contain only a single replication fork (Figure 6) that apparently originated near one end of the molecule (7). Other molecules have two forks, suggesting that replication has been initiated at both ends. In this case the daughter segments are always longer at one end than the other, perhaps reflecting the entrance of the end of the molecule into the rear zone of a replication band before the other end. Whether replication is initiated at an origin that is defined by a particular nucleotide sequence or whether initiation occurs at the ends of molecules, i.e. at the ends of telomeres is not known. Macronuclear DNA molecules do contain sequences that serve as origins of replication in yeast, which may provide an opportunity to define precisely the molecular structure present at the point of initiation of replication. 


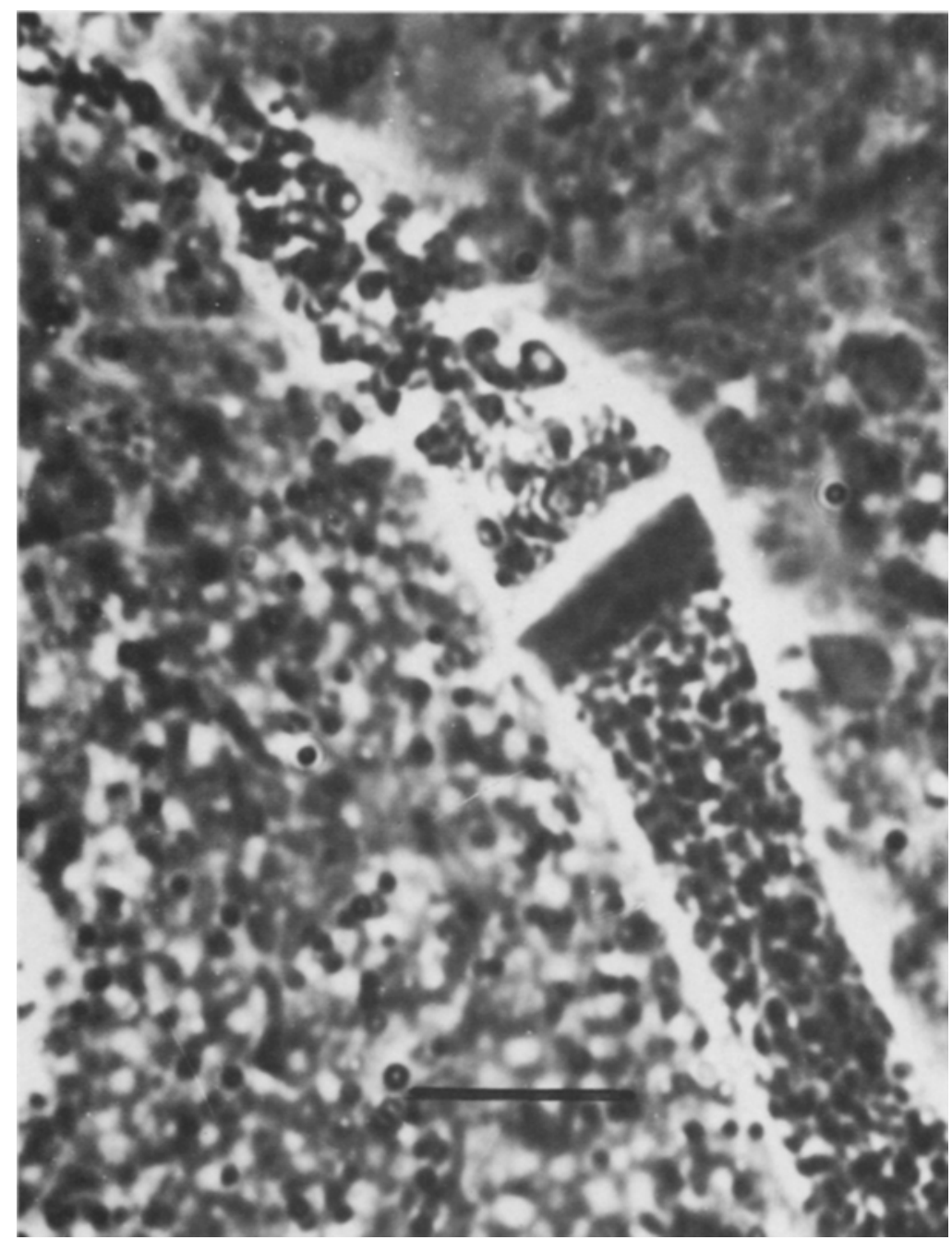

Figure 4. Phase contrast micrograph of a replication band in the macronucleus in a living hypotrich, Euplotes eurystomus. The band extends across the ribbon-shaped macronucleus and is moving away from the macronuclear tip. $\operatorname{Bar}=10 \mu \mathrm{m}$.

\section{Origin of macronuclear DNA}

Hypotrichs, like other ciliated protozoans, are capable of mating. The micronuclei in a mating pair of cells undergo meiosis, and the two cells exchange haploid micronuclei. An exchanged micronucleus fuses with a stationary micronucleus in each cell producing a new, diploid micronucleus. Unused haploid micronuclei degenerate, as does the macronucleus. The new diploid micronucleus divides mitotically without cell division, and one of the daughter micronuclei develops into a new macronucleus.
Macronuclear development is a complex process that takes about four days to complete. It begins with multiple rounds of DNA replication in a micronucleus, which results in the formation of polytene chromosomes (Figure 7$)(1,2)$. The polytene chromosomes remain only transiently and are broken down by material that transects every interband. Transection is rapidly followed by formation of vesicles, each vesicle containing the material previously contained in a band plus part of the two adjacent interbands.

At this point the size of the DNA is greatly 


\section{D.M. PrescotT: Ciliate DNA}

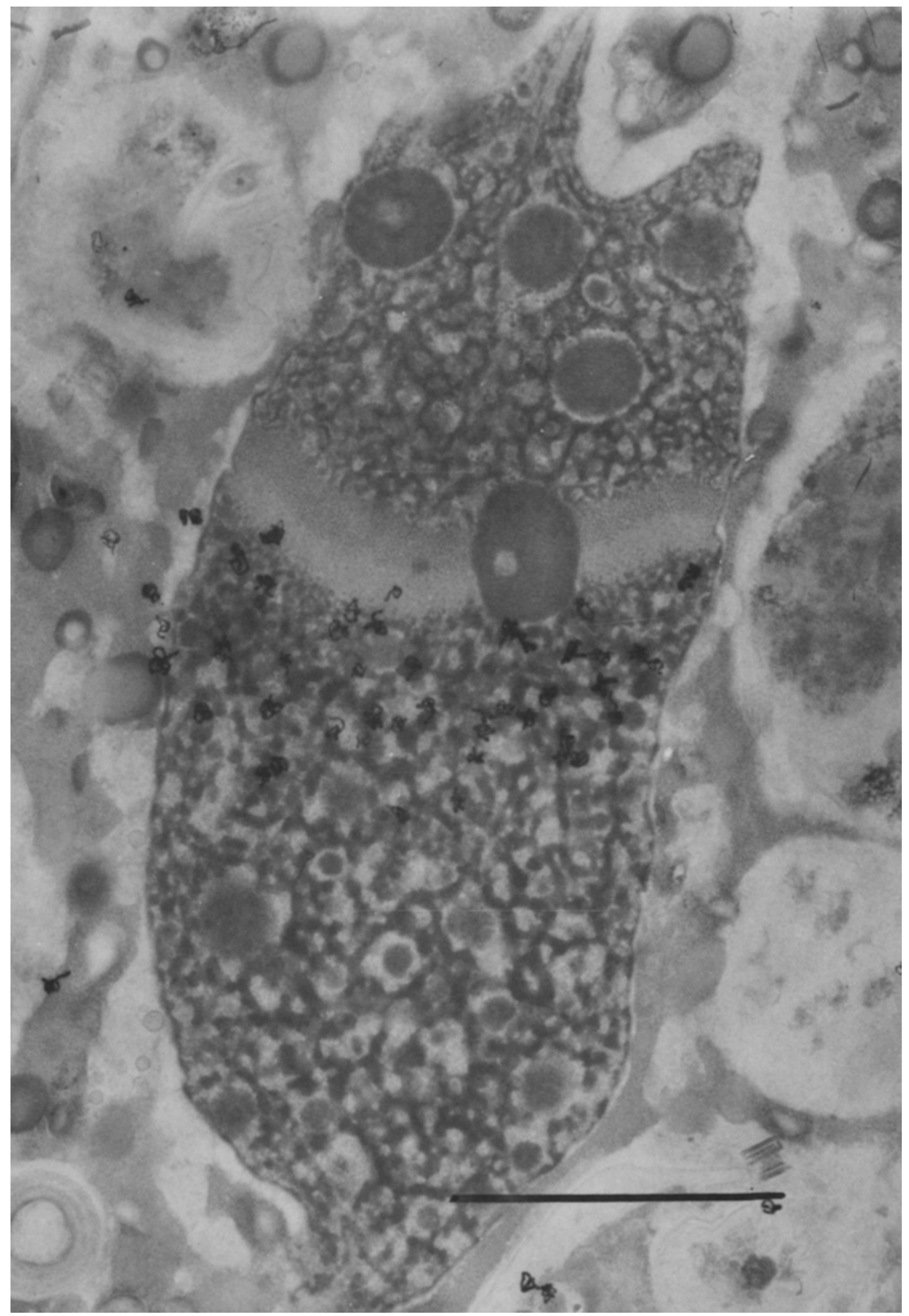


Figure 5. An electron microscope autoradiograph of the macronucleus of a hypotrich (Styx sp.) given ${ }^{3} \mathrm{H}$-thymidine. The replication band extends across the macronucleus and has two zones in it. The lighter zone is the location of DNA replication. Silver grains from incorporated ${ }^{3} \mathrm{H}$-thymidine extend from this zone back into the region of the macronucleus through which the band travelled during the ${ }^{3} \mathrm{H}$-thymidine exposure. DNA in the region ahead of the band (no silver grains) remains to be replicated. (EM by MEIYING LIN). Bar $=10 \mu \mathrm{m}$.
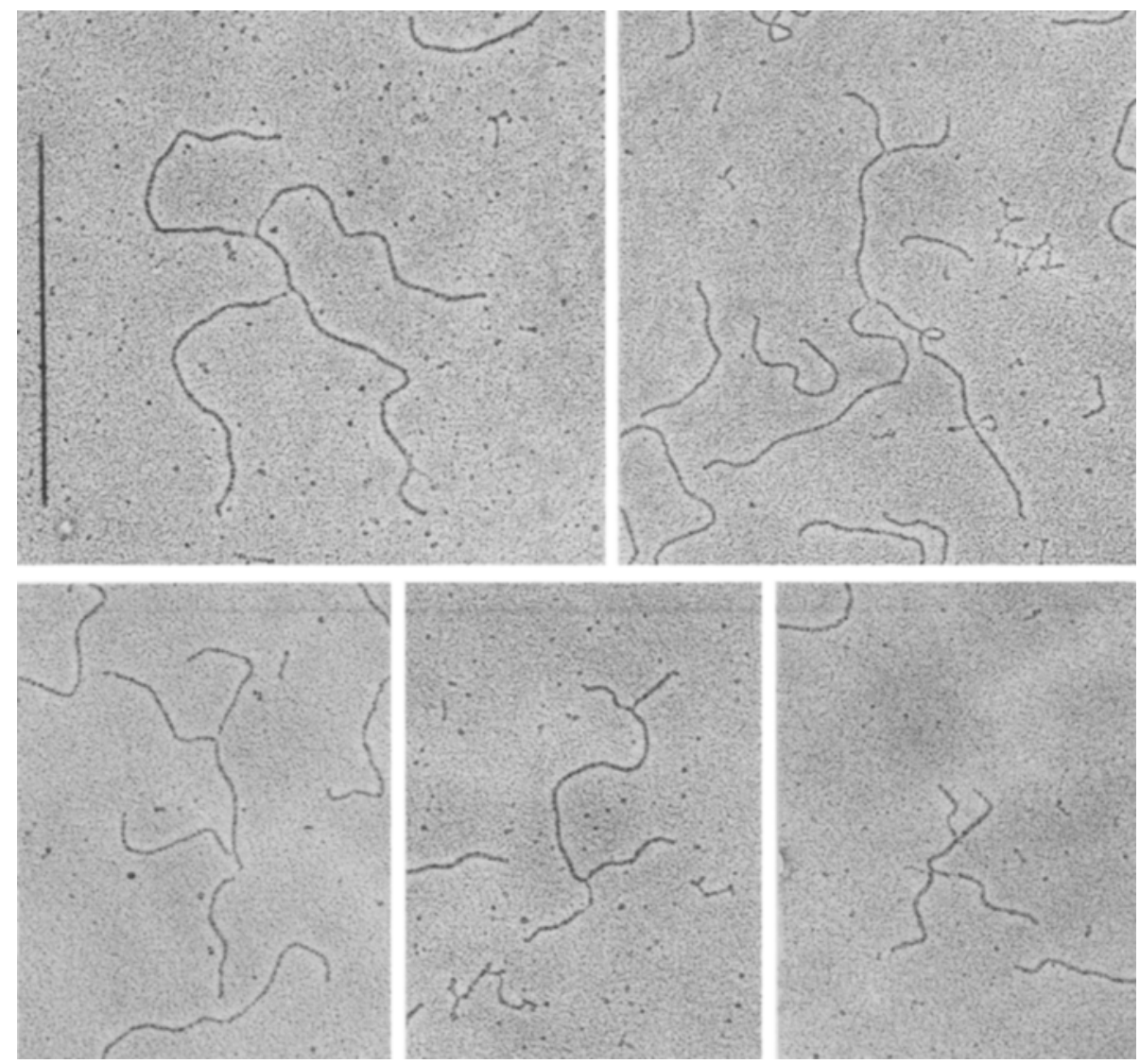

Figure 6. Replicating molecules of the hypotrich Euplotes eurystomus (by K.G. MURTI). Bar $=0.5 \mu \mathrm{m}$.

reduced from the enormously long molecules in chromosomes to much shorter molecules, but still much larger than the gene-sized molecules of the mature macronucleus (ROTH, unpublished). Within each vesicle about $95 \%$ of the DNA is degraded, leaving gene-sized molecules. From measurement of reassociation kinetics of denatured DNA we know that this destruction reduces the sequence complexity of the original chromosomal DNA by $-95 \%$. Thus, in the formation of a macronucleus the chromosomal
DNA is cut and almost completely degraded leaving small gene-sized molecules. These small molecules go through rounds of replication to produce the mature macronucleus.

These observations coupled with other studies of macronuclear and micronuclear DNA have led to the explanation shown in Figure 8. Genes are dispersed throughout micronuclear (chromosomal) DNA separated by long spacers of repetitious and unique sequences. We do not understand why polytene chromosomes are 


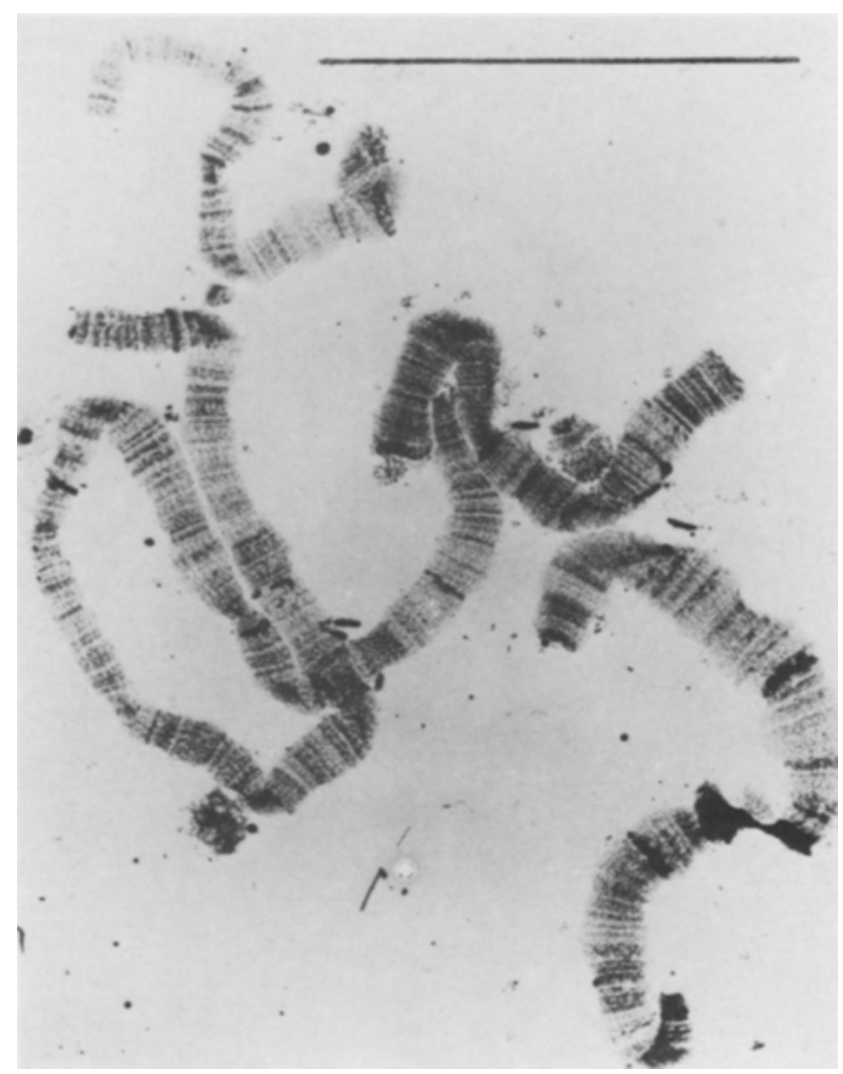

Figure 7. Four of the many polytene chromosomes in the developing macronucleus of Oxytricha nova (by K.G. MURTI). Bar $=8 \mu \mathrm{m}$.

formed to initiate macronuclear development, but in the breakdown of the chromosomes, the spacer DNA, which constitutes $\sim 95 \%$ of the total nucleotide sequence complexity of the micronuclear genome, is destroyed releasing genes. Thus, only $5 \%$ of the total chromosomal DNA is genetic in the traditional sense. The function of the DNA between genes in the chromosomes remains unknown. Studies of other eukaryotes fit the concept of large amounts of spacer DNA and small amounts of gene DNA, which provides a likely explanation for the so-called C-value or S-value paradox (10). The existence of spacer DNA allows wide differences in the total amount of DNA in the genomes of closely related species of eukaryotes with little or no change in the genes.
Two other important events occur in the excision of genes from chromosomes in hypotrichs. First, genes as they exist integrated in chromosomes do not have telomeres at their junctions with spacer DNA (5). The telomeres are added to genes after their excision. The source of the telomeric sequences is unknown. They might be synthesized de novo or derived from large banks of such sequences present at the ends of chromosomes (3).

Another profound event is the cutting and splicing of DNA to make macronuclear genes. Comparison of the sequences of a macronuclear gene with its chromosomal counterpart reveals that a gene may contain interrupting sequences that are absent from the macronuclear copy. A gene of unknown coding function, designated at 
Figure 8. A model showing destruction of chromosomal DNA and release of gene-sized molecules during development of the macronucleus in Oxytricha nova, based on cytological changes, DNA changes, and cloning of micro- and macronuclear DNAs.
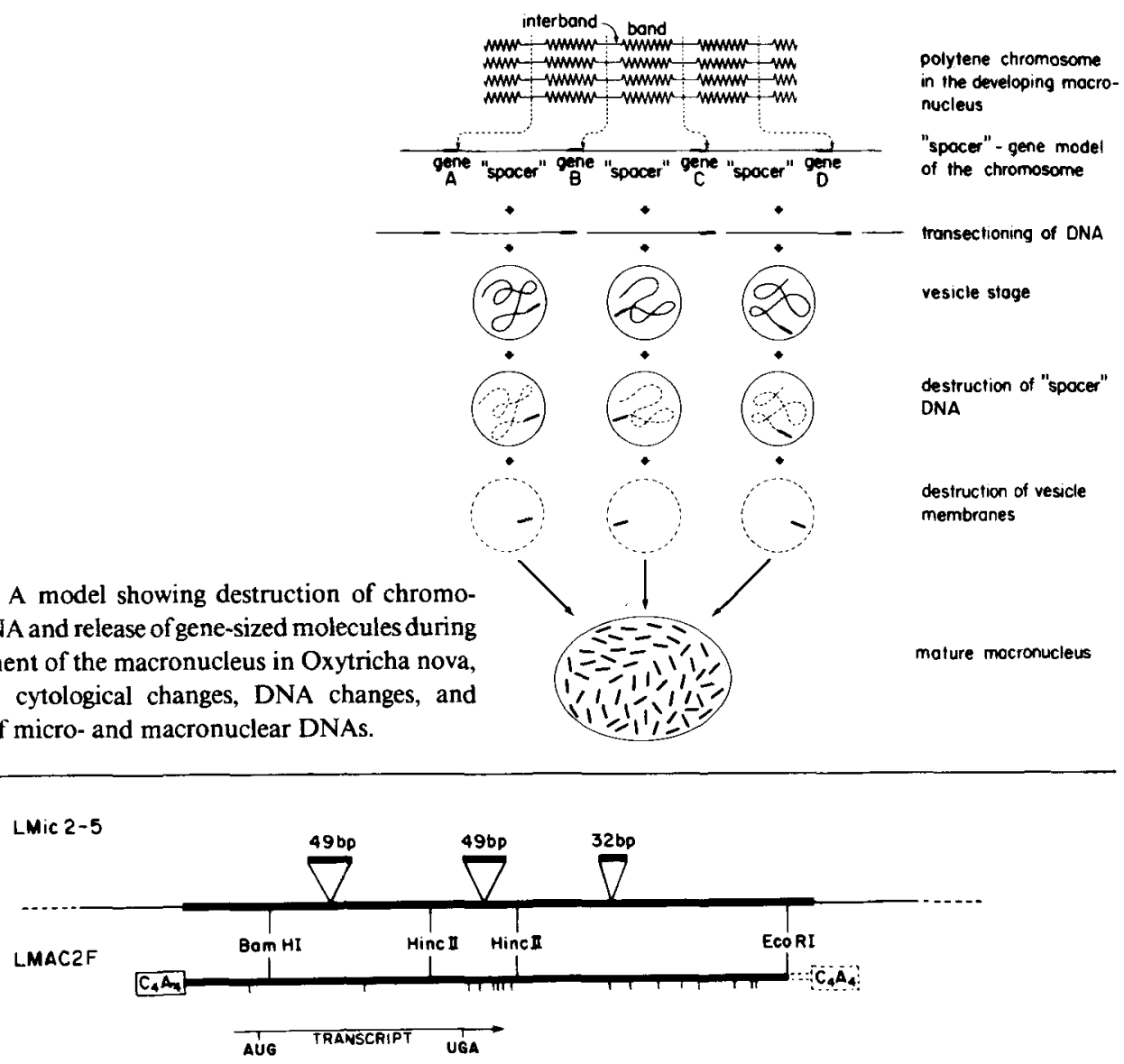

Figure 9. Diagram of a gene as it occurs in the macronucleus (LMAC2F) and in the micronucleus (LMic2-5). The macronuclear version has had telomeres added to the ends and lacks three interrupting sequences of 49,49 , and 32 base pairs present in the micronuclear version. The RNA transcripts derived from the macronuclear gene and its reading frame ( 82 amino acids) are shown.

$\mathrm{C}_{2}$ contains two 49 base pair sequences and a 32 base pair sequence that are processed out during development (Figure 9) (5). In this case four segments of DNA must be spliced together to produce the macronuclear gene. This is the most detailed example we have but other genes apparently are similarly generated.

What we have found about the hypotrichous ciliates shows us that much can be learned from them about gene and chromosome structure and function and the manipulations that organisms can exercise on their DNAs. Although the specific features may be unique to hypotrichs, the principles that they illustrate are likely to be present widely among eukaryotes in general.

\section{ACKNOWLEDGEMENTS}

This work is supported by National Institute of General Medical Sciences grant no. GM19199 to D.M.P.

\section{REFERENCES}

1. Alonso,P. \& J.PEREZ-Silva: Giant chromosomes in protozoa. Nature 205, 313-314 (1965)

2. AmmermanN, D.: Morphology and development of the macronuclei of the ciliates Stylonychia mytilus and Euplotes aediculatus. Chromosoma 33, 209-238 (1971)

3. Dawson, D. \& G. HeRrick: Telomeric properties of $\mathrm{C}_{4} \mathrm{~A}_{4}$-homologous sequences in micronuclear DNA of Oxytricha fallax. Cell 36, 171-177 (1984) 
4. Klobutcher, L.A., M.T. Swanton, P. Donini \& D.M. PRESCOTT: All gene-sized DNA molecules in four species of hypotrichs have the same terminal sequence and an unusual 3' terminus. Proc. Natl. Acad. Sci. USA 78, 3015-3019 (1981)

5. KLobutcher, L.A., C.L. JahN \& D.M. Prescott: Internal sequences are eliminated from genes during macronuclear development in the ciliate protozoan Oxytricha nova. Cell, in press (1984)

6. Lauth, M.R., B.B. Spear. J. HeumanN \& D.M. PRESCOTT: DNA of ciliated protozoa: DNA sequence diminution during macronuclear development of Oxytricha. Cell 7, 67-74 (1976)

7. Murti,K.G. \& D.M.PrescotT: Replication forms of the gene-sized DNA molecules of hypotrichous ciliates. Molec. Cell. Biol. 3, 1562-1566 (1983)

8. OKa, Y.. S. Shiota, S. Nakai. Y. Nishida \& S. OкUво: Inverted terminal repeat sequence in the macronuclear DNA of Stylonychia pustulata. Gene 10, 301-306 (1980)

9. Pluta, A.F., G.M. Dani, B.B. Spear \& V.A. ZAKIAN: Elaboration of telomeres in yeast: Recogni- tion and modification of termini from Oxytricha macronuclear DNA. Proc. Natl. Acad. Sci. USA, in press (1984)

10. PRescott, D.M.: The C-value paradox and genes in ciliated protozoa. (Alan R. Liss, Inc., New York) Mod. Cell Biol. 2, 329-352 (1983)

11. Prescott. D.M., C.J. Bostock, K.G. Murti. M.R. LAUTH \& E. GaMOW: DNA of ciliated protozoa. I. Electron microscopic and sedimentation analyses of macronuclear and micronuclear DNA of Stylonychia mytilus. Chromosoma 34, 355-366 (1971)

12. Swanton, M.T.. A.F. Greslin \& D.M. Prescott: Arrangement of coding and non-coding sequences in the DNA molecules coding for rRNAs in Oxytricha sp. Chromosoma 77, 203-215 (1980)

13. Swanton, M.T., J.M. Heumann \& D.M. PreSCOT: Gene-sized molecules of the macronuclei in three species of hypotrichs: Size distributions and absence of nicks. Chromosoma 77, 217-227 (1980) 